\title{
GRP75-mediated upregulation of HMGA1 stimulates stage I lung adenocarcinoma progression by activating the JNK/c-JUN signaling
}

\section{Guo-Bing Qiao}

Army Medical University

\section{Ren-Tao Wang}

Chinese PLA General Hospital

\section{Shu-Nan Wang}

Army Medical University

Shao-Lin Tao

Army Medical University

Qun-You Tan ( $\square$ tanqy001@163.com )

Army Medical University

Hua Jin ( $\square$ jinhua12001@tmmu.edu.cn )

Army Medical University https://orcid.org/0000-0002-1988-8009

Primary research

Keywords: HMGA1, GRP75, stage I LUAD, recurrence

Posted Date: February 24th, 2021

DOl: https://doi.org/10.21203/rs.3.rs-246900/v1

License: (9) (i) This work is licensed under a Creative Commons Attribution 4.0 International License.

Read Full License 


\section{Abstract}

Background Recurrence is a major challenge in early-stage lung adenocarcinoma (LUAD) treatment. But the mechanism is largely unknown. Here, we investigated the role and mechanism of high-mobility group AT-hook 1 (HMGA1) and glucose-regulated protein 75-kDa (GRP75) in stage I LUAD and evaluated their potential as biomarkers for predicting the recurrence and prognosis of stage I LUAD. Methods The Cancer Genome Atlas (TCGA) dataset was used to investigate the clinical significance of HMGA1 and GRP75 in early-stage LUAD. Western blotting and immunohistochemistry were used to measure protein expression levels. The biological functions of HMGA1 and GRP75 in LUAD were investigated both in vitro and in vivo through overexpression and knockdown experiments. The interaction and regulation between HMGA1 and GRP75 were evaluated with coimmunoprecipitation and ubiquitination assays. The downstream signaling pathway of the GRP75/HMGA1 axis was investigated by mRNA-sequencing analysis. Results Both HMGA1 expression levels and GRP75 expression levels were associated with recurrence in stage I LUAD patients. In particular, HMGA1 had potential as an independent prognostic factor in stage I LUAD patients. Overexpression of GRP75 or HMGA1 significantly stimulated LUAD cell growth and metastasis, while silencing GRP75 or HMGA1 inhibited LUAD cell growth and metastasis in vitro and in vivo. Importantly, GRP75 inhibited ubiquitination-mediated HMGA1 degradation by directly binding to HMGA1, thereby causes HMGA1 upregulation in LUAD. In addition, the GRP75/HMGA1 axis played its role by activating JNK/C-JUN signaling in LUAD. Conclusions The activation of GRP75/HMGA1/JNK/c-JUN signaling is an important mechanism that promotes the progression of stage I LUAD, and a high level of HMGA1 is a novel biomarker for predicting recurrence and a poor prognosis in stage I LUAD patients.

\section{Background}

Lung cancer is the most malignant human cancer with the highest incidence and mortality worldwide[1]. Lung adenocarcinoma (LUAD) is a type of non-small cell lung cancer (NSCLC) that accounts for $40 \%$ of all lung malignancies[2] and surgery is the major treatment method for patients with early-stage LUAD[3, 4]. However, $30 \% \sim 40 \%$ of stage I LUAD patients ultimately die because of locoregional or metastatic recurrence after surgery[5]. Unfortunately, no biomarker can accurately predict postoperative recurrence in patients with early-stage LUAD, and the postoperative progression mechanism of early-stage LUAD is currently unclear.

High-mobility group AT-hook 1 (HMGA1) is an architectural transcription factor, plays roles through interacting with a wide range of genes[6] and is highly expressed in a variety of cancers, including earlystage lung cancer[7-11]. Notably, increased expression of HMGA1 significantly correlated with the malignant status and prognosis of NSCLC[11]. Interestingly, although it is not lung cancer, recent studies have shown that increased expression of HMGA1 is closely related to the recurrence of various cancers, like hepatocellular carcinoma[12], cholangiocarcinoma[13], and acute lymphoblastic leukemia[14], indicating that elevated HMGA1 plays an important role in cancer recurrence. Together, these findings suggest that elevated HMGA1 may be involved in the progression of early-stage lung cancer. However, the 
upregulation mechanism and the functional role of HMGA1 in the progression of early-stage LUAD have not been reported.

Glucose-regulated protein 75 (GRP75) is a molecular chaperon belonging to the heat shock protein 70 family and implicated in multiple biological functions, like stress responses[15], and differentiation[16], Increased expression of GRP75 has been reported in several cancers, including NSCLC[17], leukemia[18], brain cancer[19], colorectal adenocarcinoma[20], and hepatocellular carcinoma[21]. Notably, previous studies showed that high expression of GRP75 was closely correlated with these cancers progression. For example, according to Yi et al. reported that increased expression GRP75 was significantly correlated with early tumor recurrence in patients with liver cancer[22], according to Sun et al. reported that GRP75 overexpression predicted a poor prognosis in patients with early-stage NSCLC[17]. However, the correlation between GRP75 expression and early-stage LUAD recurrence have not been reported. Additionally, the mechanism of GRP75 promotes the early-stage LUAD progression is not clear.

In this study, we reported that overexpression of GRP75 and HMGA1 were closely correlated with recurrence in stage I LUAD patients. Notably, overexpression of HMGA1 was an independent prognostic indicator for stage I LUAD patients. In addition, we demonstrated that GRP75 protected HMGA1 from ubiquitination-mediated protein degradation by directly binding to HMGA1, thereby, causes upregulation of HMGA1 in LUAD. Additionally, we identified that GRP75 played a tumor-promoting role depending on HMGA1 in LUAD. Finally, we demonstrated that the GRP75/HMGA1 axis promoted LUAD progression by activating JNK/c-JUN signaling.

\section{Materials And Methods}

\section{Materials}

Fetal bovine serum (FBS) and cell culture medium were purchased from HyClone (South Logan, UT, USA). MG132 and kanamycin were purchased from Sigma (St. Louis, MO, USA). JNK IN8 was obtained from MedChem Express (Shanghai, China). Plasmids for overexpressing HMGA1 or GRP75 and HMGA1specific short hairpin RNA (shRNA) were designed and synthesized by GeneChem (Shanghai, China). Small interfering RNA (siRNA) oligonucleotides targeting HMGA1 or GRP75 were obtained from RiboBio (Guangzhou, China). A cell counting kit-8 (CCK8) kit was purchased from Bioss (Beijing, China). A migration assay kit was obtained from BD Biosciences (Franklin Lakes, NJ, USA). Cell invasion assay chambers (24 wells) were purchased from Corning (NY, USA). Cycloheximide (CHX) and primary antibodies against HMGA1, GRP75, Ki67, Ubiquitin, JNK, P-JNK (Thr183/Tyr185), c-JUN, P-C-JUN (Ser63) and P-C-JUN (Ser73) and GAPDH were purchased from Cell Signaling Technology (Danvers, MA, USA). A Pierce coimmunoprecipitation kit was obtained from Thermo Fisher Scientific (Waltham, MA, USA).

\section{Cell lines, cell transfection and RNA interference}

The lung adenocarcinoma cell lines A549 and PC9 were obtained from the American Type Culture Collection (ATCC; Manassas, VA, USA). All cell lines were maintained in Dulbecco's modified Eagle's 
medium supplemented with $10 \% \mathrm{FBS}$ at $37^{\circ} \mathrm{C}$ in a humidified atmosphere with $5 \% \mathrm{CO}_{2}$.

Cells were transfected with the indicated plasmids or siRNAs using Lipofectamine 3000 (Invitrogen, CA) according to the manufacturer's protocol. The siRNA sequences against HMGA1 were as follows: 5'GAAGTGCCAACACCTAAGA-3' and 5'-AGCGAAGTGCCAACACCTA-3'. The siRNA sequences against GRP75 were as follows: 5'-TGCCTATGGTCTAGACAAA-3' and 5'-GGATTGTCACTGATCTAAT-3'. The control siRNA sequence was 5'-UUCUCCGAACGUGUCACGUTT-3'. The HMGA1-specific short hairpin RNA sequence was 5'-GATCCCCTGCTACCAGCGCCAAATGTTCTCGAGAACATTTGGCGCTGGTAGCAGTTTTTGGAT-3', and the control sequence was 5'-TTCTCCGAACGTGTCACGT-3'. After transfection for 48 or 72 hours, the cells were subjected to further analyses.

\section{Transwell and cell viability assays}

The migratory and invasive abilities of LUAD cells were assessed with a transwell assay. The indicated cells were transfected with the indicated oligonucleotides or plasmids for $48 \mathrm{~h}$. Then, $3 \times 10^{4}$ cells in serum-free growth medium were seeded in the upper chambers. The lower chambers were filled with the same medium supplemented with 10\% FBS. After $24 \mathrm{~h}$, the cells that had invaded the lower side of the chamber were fixed with $4 \%$ paraformaldehyde (PFA), stained with $0.1 \%$ crystal violet and dried. The number of invading cells was photographed and counted using an Olympus inverted microscope at 100 magnification.

For cell viability assays, the indicated cells were plated in 96 -well plates at $4 \times 10^{3}$ cells per well. At the indicated time points, cell viability was estimated by using CCK8 reagent according to the manufacturer's instructions. Absorbance was measured at a wavelength of $450 \mathrm{~nm}$ by using a microplate reader.

\section{Colony formation assays}

After $48 \mathrm{~h}$ of transfection, cells were trypsinized and resuspended in $0.5 \mathrm{ml} 0.35 \%$ agar in growth medium at a density of 1000 cells/well (24-well plate). Then, the agar-cell mixture was plated on top of a solid layer of $0.8 \%$ agar in the growth medium. The cells were cultured until visible clones appeared (approximately 3 weeks). The cell clones were washed twice with PBS, fixed with methanol for 20 mins, stained with $0.1 \%$ crystal violet for $1 \mathrm{~h}$, washed with PBS, and counted.

\section{Western blot analysis}

Western blot analysis was carried out according to a standard protocol. Cell lysates were generated from cultured cells with RIPA lysis buffer containing protease inhibitors (Roche). The protein concentration was determined by using a Bradford kit (Pierce, Thermo Fisher Scientific). Equal amounts of total protein were loaded, separated by SDS-PAGE and then transferred to PVDF membranes (Bio-Rad, Hercules, CA, USA). After blocking in $5 \%$ nonfat milk, the membranes were incubated overnight at $4{ }^{\circ} \mathrm{C}$ with appropriate primary antibodies and then with secondary antibodies conjugated to horseradish peroxidase for 2 hours at room temperature. After washing, the bands of interest were analyzed by using an ECL kit. Images 
were acquired using the Bio-Spectrum Gel Imaging System (Bio-Rad, USA). Then, quantification of the Western blot results was performed using Image Lab software, and the bands were normalized with GAPDH as the internal control.

\section{Immunoprecipitation and mass spectrometry}

Immunoprecipitation was performed using a Thermo Fisher Scientific Pierce coimmunoprecipitation kit following the manufacturer's protocol. Briefly, the indicated antibodies were first immobilized for $2 \mathrm{~h}$ using AminoLink Plus coupling resin. The resin was then washed and incubated with lysate overnight at $4^{\circ} \mathrm{C}$. After the incubation, the resin was washed, and proteins were eluted using elution buffer. A negative control that was provided with the co-IP kit to assess nonspecific binding received the same treatment as the samples with IgG. The immunoprecipitated samples were resolved by SDS-PAGE, followed by Western blotting with appropriate antibodies. For liquid chromatography-mass spectrometry (LC-MS) analysis, samples, which included those with HMGA1 overexpression and a control, were separated using electrophoresis, and specific bands were identified using a mass spectrometry system; the molecules corresponding to the bands were identified in a human proteomic library to obtain proteins directly bound to HMGA1.

\section{Degradation and deubiquitylation assay}

To detect HMGA1 degradation mediated by GRP75 in A549 cells, cells with or without GRP75 overexpression were treated with $\mathrm{CHX}(\mathrm{CST}, 100 \mu \mathrm{g} / \mathrm{ml})$ for the indicated durations before analysis. Then, cell lysates were analyzed using Western blotting with an anti-HMGA1 antibody. To detect HMGA1 deubiquitination in A549 cells, cells were transfected with the indicated plasmids and treated with $20 \mu \mathrm{M}$ proteasome inhibitor MG132 (Sigma) for $8 \mathrm{~h}$ before harvest. Then, the cells were lysed in IP lysis/wash buffer and incubated with the anti-HMGA1 antibody, which was used for IP. The immunoprecipitated samples were resolved by SDS-PAGE, followed by Western blotting with an anti-ubiquitin antibody.

\section{Bioinformatic analysis}

Publicly available databases were used to evaluate gene expression and predict potential mechanisms. A clinical dataset of stage I LUAD patients was obtained from the TCGA database and analyzed using TIMER[23]. mRNA-sequencing analysis was performed by Huada Genomics Institute (BGI, Guangzhou, China), and KEGG pathway analysis was conducted with the Dr. Tom program (https://biosys.bgi.com).

\section{Animal experiments}

Animal studies were conducted at Daping Hospital, according to the National Institutes of Health Guide for the Care and Use of Laboratory Animals. All animal experiments were approved by the Animal Research Committee of Army Medical University. Six-week-old female nude mice were utilized. To investigate the effects of oncogenic HMGA1 on LUAD cell tumorigenicity, the indicated cells were transfected with HMGA1 or HMGA1-specific shRNA-expressing or control plasmids. After $72 \mathrm{~h}$ of 
transfection, $2 \times 10^{6}$ cells in $0.1 \mathrm{~mL}$ PBS were injected subcutaneously (five mice per group). Tumor length $(\mathrm{L})$ and width $(\mathrm{W})$ were measured weekly, and tumor volume $(\mathrm{V})$ was calculated using the formula $V=L \times$ $\mathrm{W}^{2} / 2$. Four weeks after cell injection, these mice were sacrificed, and the tumors were harvested and weighed. To investigate the presence of Ki67 proteins in mouse tumor tissues, an immunohistochemistry assay was performed as described previously[24]. For metastatic ability experiments, four groups of five mice each were given tail vein injections of $2 \times 10^{6}$ the indicated cells, respectively. Four weeks after injection, all mice were sacrificed, the tumor nodules formed on the lung surface were counted. The lungs were embedded with paraffin and sliced for HE staining.

\section{Statistical analysis}

All data are presented as the mean $\pm \mathrm{SD}$, and statistical analyses were performed using GraphPad Prism 8 (GraphPad Software, Inc., La Jo Ila, CA, USA). The statistical significance of differences between treatment groups was analyzed by a two-tailed paired t-test or one-way analysis of variance (ANOVA). The survival rate was calculated using Kaplan-Meier survival analysis. $P<0.05$ was considered statistically significant.

\section{Results}

\section{Upregulated expression of HMGA1 is associated with recurrence and a poor prognosis in stage I LUAD patients}

First, we used a TCGA dataset to investigate whether the expression level of HMGA1 is involved in the recurrence of stage I LUAD. As in previous reports, the TCGA analysis results showed that HMGA1 expression was abnormally upregulated in many types of cancer tissue, including LUAD tissue, compared to the corresponding normal tissue (Fig. 1a, b). Notably, the expression level of HMGA1 in stage I LUAD tissues was significantly higher than that in normal lung tissues (Fig. 1C), and the high expression level of HMGA1 in primary tumors was closely correlated with recurrence in patients with stage I LUAD (Fig. 1d). In addition, the TCGA dataset analysis results showed that the stage I LUAD patient group with a high level of HMGA1 had a shorter median recurrence-free survival (RFS) time (Fig. 1e) and lower overall survival (OS) rate than the low HMGA1 expression group (Fig. 1f). Collectively, these results indicate that the increased expression level of HMGA1 is closely correlated with recurrence and a poor prognosis in stage I LUAD patients.

\section{HMGA1 stimulates LUAD progression and is a therapeutic target in LUAD}

Next, we investigated whether the upregulated expression of HMGA1 directly involved in stimulation of LUAD progression. We first generated a construct expressing green fluorescent protein (GFP)-tagged HMGA1 and transfected it into LUAD cells (Fig. 2a), then performed cell viability, soft agar colony formation and transwell assays. Our results showed that overexpression of HMGA1 significantly stimulated cell viability (Fig. 2b), soft agar colony formation (Fig. 2c), migration and invasion (Fig. 2d) in both A549 cells and PC9 cells. These in vitro results were further confirmed in vivo using animal models. 
As shown in Fig. $2 e$ and $2 f$, subcutaneous xenograft model experiment shows the tumor growth rate and tumor weight in HMGA1 overexpression group were higher than control group. Additionally, an increased expression level of the cell proliferation marker protein Ki67 was detected in HMGA1-overexpressing tumor tissues (Fig. 2g). In addition, lung metastasis model experiment shows the mice in the HMGA1 group had more and larger lung metastatic nodules compared to the vector control (Fig. $2 \mathrm{~h}$, i). These findings suggest that upregulated expression of HMGA1 directly stimulate LUAD progression.

These findings prompted us to investigate whether HMGA1 silencing can suppress LUAD progression. As expected, silencing of HMGA1 (Fig. 3a) significantly suppressed LUAD cell viability (Fig. 3b), soft agar colony formation (Fig. 3c), migration and invasion in vitro (Fig. 3d). Consistently, animal experiments also showed that silencing HMGA1 expression (Additional file 2: Fig S1) dramatically suppressed tumor growth (Fig. 3e, f), tumor cell proliferation (Fig. 3g) and metastasis (Fig. 3h, i). These findings indicate that inhibition of HMGA1 may be a useful strategy for treating LUAD.

\section{Upregulated protein expression of HMGA1 was caused by increased expression of GRP75 in LUAD}

To elucidate the regulatory and functional mechanism of HMGA1 overexpression in LUAD, we identified proteins that bind to HMGA1 in LUAD cells using IP/LC-MS assays. Our results identified 25 proteins that might specifically interact with HMGA1 in LUAD cells (Fig. 4a, b, Additional file 1: Table S1). Among them, GRP75 was selected for further study, as GRP75 was previously reported to promote metastasis and be closely associated with early recurrence in liver cancer[25]. The interaction between GRP75 and HMGA1 in LUAD was further confirmed using coimmunoprecipitation (co-IP) assays (Fig. 4c, Additional file 3: Fig S2). Notably, TCGA data analyses showed that GRP75 protein levels were positively correlated with HMGA1 protein levels in stage I LUAD tissues (Fig. 4d). Since the role of GRP75 in early-stage LUAD is not clear, we then investigated the effect of GRP75 on early-stage LUAD using a TCGA dataset analysis and in vitro experiments. The TCGA dataset analysis showed that GRP75 was highly expressed in stage I LUAD tissues compared to normal tissues (Fig. 4e) and that high expression levels of GRP75 were closely associated with recurrence (Fig. 4f) and shortened RFS in patients with stage I LUAD (Fig. 4g). In addition, the in vitro experiments showed that overexpression of GRP75 significantly stimulated LUAD cell viability (Fig. 4h), migration and invasion (Fig. 4i), while silencing GRP75 significantly suppressed LUAD cell viability (Fig. 4j), migration and invasion (Fig. 4k). These findings suggest that GRP75 acts as an oncoprotein in early-stage LUAD and may be a partner protein of HMGA1.

Next, we investigated how HMGA1 and GRP75 affect each other's expression and functions in LUAD cells. Our Western blot data showed that HMGA1 overexpression or silencing did not affect GRP75 protein levels, while GRP75 overexpression or silencing upregulated or downregulated HMGA1 protein levels in LUAD cell lines, respectively (Fig. 5a). In addition, in vitro experiments showed that silencing of HMGA1 significantly inhibited the GRP75 overexpression-induced stimulation of LUAD cell viability (Fig. 5b), invasion and migration (Fig. 5c). Interestingly, multivariate analysis identified HMGA1 but not GRP75 as an independent prognostic predictor in stage I LUAD (Table 1). Importantly, RFS analysis showed that there was no correlation between a high expression level of GRP75 and recurrence in stage I LUAD 
patients with low expression of HMGA1 (Fig. 5d). Taken together, these findings suggest that HMGA1 is a downstream protein of GRP75 and that GRP75 promotes HMGA1-dependent early-stage LUAD progression.

Further, we investigated the mechanism by which GRP75 upregulates HMGA1 expression in LUAD. Unlike our protein data, our mRNA data showed that the mRNA expression level of HMGA1 was not affected by GRP75 overexpression or silencing (Fig. 5e), suggesting that GRP75 regulates HMGA1 expression through posttranscriptional regulation. Because GRP75 binds directly to HMGA1, we speculated that GRP75 may participate in the regulation of HMGA1 protein degradation. To confirm this hypothesis, we investigated the effect of GRP75 on the half-life of the HMGA1 protein in LUAD cells treated with the protein synthesis inhibitor cycloheximide (CHX). Compared with the vector control, GRP75 overexpression robustly prolonged the half-life of the endogenous HMGA1 protein in A549 cells (Fig. 5f). In addition, the ubiquitination of HMGA1 in A549 cells was dramatically inhibited by GRP75 overexpression (Fig. 5g). These findings suggest that GRP75 upregulates the HMGA1 protein level by protecting the HMGA1 protein from ubiquitination-mediated degradation.

\section{GRP75/HMGA1 axis stimulates LUAD progression by activating the JNK/c-JUN signaling pathway}

To further investigate the molecular mechanisms by which the GRP75/HMGA1 axis stimulates the progression of early-stage LUAD, we performed mRNA sequencing using HMGA1- or GRP75overexpressing LUAD cells and their corresponding control cells (Fig. 6a). Then, KEGG signaling pathway enrichment analysis was performed using a set of genes that similarly upregulated or downregulated in cells with overexpression of HMGA1 and GRP75 compared to their corresponding control. As shown in Fig. $6 \mathrm{~b}$, we found that the JNK signaling pathway was correlated with overexpressed HMGA1 and GRP75 in LUAD. In addition, our Western blot analysis showed that overexpression of HMGA1 stimulated the phosphorylation of JNK (Thr183/Tyr185) and its downstream protein c-JUN (Ser63 and Ser73) in LUAD cells, whereas HMGA1 silencing inhibited the phosphorylation of both JNK (Thr183/Tyr185) and c-JUN (Ser63 and Ser73) (Fig. 6c). Consistent with the HMGA1 results, overexpression of GRP75 also increased the phosphorylation of both JNK and c-JUN in LUAD cells, and the upregulation of phosphor-JNK and phosphor-c-JUN levels induced by GRP75 overexpression was blocked by silencing HMGA1 (Fig. 6d). Notably, treatment with the JNK inhibitor JNK IN8 effectively blocked GRP75- and/or HMGA1overexpression inhibited cell viability (Fig. 6e), migration and invasion (Fig. 6f). Collectively, these results reveal that the GRP75/HMGA1 axis promotes LUAD progression by activating the JNK/C-JUN signaling pathway (Fig. 6g).

\section{Discussion}

Here, we report for the first time that upregulated expression levels of both GRP75 and HMGA1 are closely correlated with recurrence in stage I LUAD patients. In particular, HMGA1 has great potential as an independent biomarker for predicting the recurrence and poor prognosis of stage I LUAD patients. Our results are supported by research from other groups. Although not in LUAD, similar results have been 
obtained in other tumors. According to Yi et al., a high expression level of GRP75 is closely correlated with liver cancer early recurrence[25]. According to Liu et al., high expression of HMGA1 is closely correlated with glioblastoma recurrence[26]. However, the sample size used in this study is limited, so the finding needs to be further verified in a larger clinical sample before clinical use.

Then, we also clarified the mechanism of HMGA1 upregulation in LUAD. GRP75 is overexpressed in several cancers [27] and involved in posttranslational modification of some transcription factors, especially ubiquitinylation[28]. For instance, GRP75 stabilizes the proproliferative association of Hsp90 and epidermal growth factor receptor by rescuing the Tid1L-promoted ubiquitinylation and degradation of epidermal growth factor receptor, thereby contributing to counteracting death pathways[29]. In this study, our in vitro data showed that GRP75 upregulated HMGA1 protein levels and GRP75 protect HMGA1 from ubiquitination-mediated protein degradation by directly binding to HMGA1. Notably, clinical data show that expression level of HMGA1 positively correlated with GRP75 expression level in stage I LUAD and GRP75 was highly expressed in stage I LUAD. Together, these findings indicating that abnormally high expressed GRP75 inhibits ubiquitination-mediated HMGA1 degradation, thereby causes HMGA1 upregulation in LUAD. However, the detailed mechanism by which GRP75 inhibits HMGA1 ubiquitination and the mechanism underlying GRP75 upregulation in stage I LUAD need further study.

Finally, we elucidated the oncogenic mechanism of GRP75 and HMGA1 in LUAD progression. The oncogenic role and therapeutic potential of GRP75 or HMGA1 have been reported in several cancers[3032]. Consistently, the present study results also show that both GRP75 and HMGA1 play oncogenic roles in LUAD and exhibit potential as therapeutic targets in LUAD. However, the highlight of this study is that we using in vitro and clinical data demonstrated for the first time that GRP75 depends on HMGA1 to play an oncogenic role in early-stage LUAD. Notably, we identified that the GRP75/HMGA1 axis plays an oncogenic role by activating JNK/c-JUN signaling. Various findings have reported that activation of the JNK/c-JUN signaling pathway is closely correlated with cancer progression, including recurrence[33-35]. For instance, Jorgense et al. reported that activation of c-JUN was associated with cell proliferation and a shortened relapse-free period in superficially spreading malignant melanoma[36]. According to Hagiwara et al., activation of c-JUN predicts a high risk of recurrence after hepatic resection for hepatocellular carcinoma[37]. To the best of our knowledge, this is the first evidence that HMGA1, as a critical target gene of GRP75, activates the JNK/c-JUN signaling pathway in the progression mechanism of lung adenocarcinoma. However, the detailed mechanism by which HMGA1 activates the JNK pathway in LUAD requires further study.

\section{Conclusions}

In summary, our study combined TCGA dataset analysis and experimental studies to establish the oncogenic roles of HMGA1 and GRP75 in stage I LUAD and the mechanism of HMGA1 upregulation. We show that high expressed GRP75 causes upregulation of HMGA1 expression by inhibiting HMGA1 ubiquitination through binding to HMGA1 in LUAD and that upregulated HMGA1 expression promotes LUAD progression by activating the JNK/c-JUN signaling pathway. Additionally, our findings suggest that 
both GRP75 and HMGA1 are useful therapeutic targets for LUAD and that a high expression level of HMGA1 is an independent biomarker for predicting recurrence and a poor prognosis in stage I LUAD.

\section{Abbreviations}

NSCLC: Non-small-cell lung cancer; LUAD: Lung adenocarcinoma; HMGA1: High-mobility group AT-hook 1; EMT: Epithelial-mesenchymal transition; CSCs: Cancer stem cells; GRP75: Glucose-regulated protein 75kDa; TCGA: The Cancer Genome Atlas; PTM: Post-translational modification; JNKs: c-JUN N-terminal kinases; MAPK: mitogen-activated protein kinase; RFS: recurrence-free survival; OS: overall survival; CCK8: cell counting kit-8; $\mathrm{CHX}$ : Cycloheximide.

\section{Declarations}

\section{Ethics approval and consent to participate}

The animal experiments in this study were approved by the Animal Research Committee of Army Medical University, according to the National Institutes of Health Guide for the Care and Use of Laboratory Animals.

\section{Consent for publication}

Not applicable.

\section{Availability of data and materials}

All data generated or analysed during this study are included in this published article.

\section{Competing interests}

The authors declare that they have no competing interests

\section{Funding}

This work was supported by the National Natural Science Foundation of China (81672283 to H.J.), the Natural Science Foundation of Chong Qing, China (cstc2015jcyjA10073 to S.T.), and the Startup Fund for Talented Scholars of Daping Hospital, Army Medical University (to H.J.).

\section{Acknowledgements}

Not applicable.

\section{Conflict of Interest}

The authors declare that they have no competing interests. 
Acknowledgements

Not applicable.

Author contributions

HJ and QYT contributed to the conception and design of this study. GBQ performed laboratory and animal experiments. GBQ, RTW, SNW, SLT and HJ analyzed the data. HJ and GBQ wrote the manuscript. All authors read and approved the final manuscript.

\section{References}

1. Herbst RS, Morgensztern D, Boshoff C: The biology and management of non-small cell lung cancer. Nature 2018, 553(7689):446-454.

2. Chen Z, Fillmore CM, Hammerman PS, Kim CF, Wong KK: Non-small-cell lung cancers: a heterogeneous set of diseases. Nat Rev Cancer 2014, 14(8):535-546.

3. Goldstraw P, Chansky K, Crowley J, Rami-Porta R, Asamura H, Eberhardt WE, Nicholson AG, Groome P, Mitchell A, Bolejack V et al: The IASLC Lung Cancer Staging Project: Proposals for Revision of the TNM Stage Groupings in the Forthcoming (Eighth) Edition of the TNM Classification for Lung Cancer. J Thorac Oncol 2016, 11(1):39-51.

4. Yoshizawa A, Motoi N, Riely GJ, Sima CS, Gerald WL, Kris MG, Park BJ, Rusch VW, Travis WD: Impact of proposed IASLC/ATS/ERS classification of lung adenocarcinoma: prognostic subgroups and implications for further revision of staging based on analysis of $\mathbf{5 1 4}$ stage I cases. Mod Pathol 2011, 24(5):653-664.

5. Brock MV, Hooker CM, Ota-Machida E, Han Y, Guo M, Ames S, Glockner S, Piantadosi S, Gabrielson E, Pridham $\mathrm{G}$ et al: DNA methylation markers and early recurrence in stage I lung cancer. $N$ Engl $J$ Med 2008, 358(11):1118-1128.

6. Reeves R: Nuclear functions of the HMG proteins. Biochim Biophys Acta 2010, 1799(1-2):3-14.

7. Sarhadi VK, Wikman H, Salmenkivi K, Kuosma E, Sioris T, Salo J, Karjalainen A, Knuutila S, Anttila S: Increased expression of high mobility group A proteins in lung cancer. The Journal of pathology 2006, 209(2):206-212.

8. Lin SY, Peng F: Association of SIRT1 and HMGA1 expression in non-small cell lung cancer. Oncology letters 2016, 11(1):782-788.

9. Wang Y, Hu L, Zheng Y, Guo L: HMGA1 in cancer: Cancer classification by location. J Cell Mol Med 2019.

10. Thanos $D$, Maniatis T: The high mobility group protein HMG $I(Y)$ is required for NF-kappa Bdependent virus induction of the human IFN-beta gene. Cell 1992, 71(5):777-789.

11. Zhang Z, Wang Q, Chen F, Liu J: Elevated expression of HMGA1 correlates with the malignant status and prognosis of non-small cell lung cancer. Tumour biology : the journal of the International Society 
for Oncodevelopmental Biology and Medicine 2015, 36(2):1213-1219.

12. Chang ZG, Yang LY, Wang W, Peng JX, Huang GW, Tao YM, Ding X: Determination of high mobility group A1 (HMGA1) expression in hepatocellular carcinoma: a potential prognostic marker. Dig Dis Sci 2005, 50(10):1764-1770.

13. Quintavalle C, Burmeister K, Piscuoglio S, Quagliata L, Karamitopoulou E, Sepe R, Fusco A, Terracciano LM, Andersen JB, Pallante $P$ et al: High mobility group A1 enhances tumorigenicity of human cholangiocarcinoma and confers resistance to therapy. Mol Carcinog 2017, 56(9):2146-2157.

14. Roy S, Di Cello F, Kowalski J, Hristov AC, Tsai HL, Bhojwani D, Meyer JA, Carroll WL, Belton A, Resar LM: HMGA1 overexpression correlates with relapse in childhood B-lineage acute lymphoblastic leukemia. Leuk Lymphoma 2013, 54(11):2565-2567.

15. Lu WJ, Lee NP, Kaul SC, Lan F, Poon RTP, Wadhwa R, Luk JM: Mortalin-p53 interaction in cancer cells is stress dependent and constitutes a selective target for cancer therapy. Cell Death and Differentiation 2011, 18(6):1046-1056.

16. Xu J, Xiao HH, Sartorelli AC: Attenuation of the induced differentiation of HL-60 leukemia cells by mitochondrial chaperone HSP70. Oncol Res 1999, 11(9):429-435.

17. Sun J, Che SL, Piao JJ, Xu M, Chen LY, Lin ZH: Mortalin overexpression predicts poor prognosis in early stage of non-small cell lung cancer. Tumor Bio/2017, 39(3).

18. Pizzatti L, Sa LA, de Souza JM, Bisch PM, Abdelhay E: Altered protein profile in chronic myeloid leukemia chronic phase identified by a comparative proteomic study. Biochim Biophys Acta 2006, 1764(5):929-942.

19. Takano S, Wadhwa R, Yoshii Y, Nose T, Kaul SC, Mitsui Y: Elevated levels of mortalin expression in human brain tumors. Exp Cell Res 1997, 237(1):38-45.

20. Rozenberg P, Kocsis J, Saar M, Prohaszka Z, Fust G, Fishelson Z: Elevated levels of mitochondrial mortalin and cytosolic HSP70 in blood as risk factors in patients with colorectal cancer. International Journal of Cancer 2013, 133(2):514-518.

21. Wadhwa R, Takano S, Kaur K, Deocaris CC, Pereira-Smith OM, Reddel RR, Kaul SC: Upregulation of mortalin/mthsp70/Grp75 contributes to human carcinogenesis. International Journal of Cancer 2006, 118(12):2973-2980.

22. Yi X, Luk JM, Lee NP, Peng JR, Leng XS, Guan XY, Lau GK, Beretta L, Fan ST: Association of mortalin (HSPA9) with liver cancer metastasis and prediction for early tumor recurrence. Mol Cell Proteomics 2008, 7(2):315-325.

23. Li T, Fan J, Wang B, Traugh N, Chen Q, Liu JS, Li B, Liu XS: TIMER: A Web Server for Comprehensive Analysis of Tumor-Infiltrating Immune Cells. Cancer research 2017, 77(21):e108-e110.

24. Xu CX, Jere D, Jin H, Chang SH, Chung YS, Shin JY, Kim JE, Park SJ, Lee YH, Chae CH et al: Poly(ester amine)-mediated, aerosol-delivered Akt1 small interfering RNA suppresses lung tumorigenesis. American journal of respiratory and critical care medicine 2008, 178(1):60-73.

25. Yi X, Luk JM, Lee NP, Peng J, Leng X, Guan XY, Lau GK, Beretta L, Fan ST: Association of mortalin (HSPA9) with liver cancer metastasis and prediction for early tumor recurrence. Mol Cell Proteomics 
2008, 7(2):315-325.

26. Liu B, Pang B, Liu H, Arakawa Y, Zhang R, Feng B, Zhong P, Murata D, Fan H, Xin T et al: High mobility group A1 expression shows negative correlation with recurrence time in patients with glioblastoma multiforme. Pathology, research and practice 2015, 211(8):596-600.

27. Wadhwa R, Takano S, Kaur K, Deocaris CC, Pereira-Smith OM, Reddel RR, Kaul SC: Upregulation of mortalin/mthsp70/Grp75 contributes to human carcinogenesis. International journal of cancer 2006, 118(12):2973-2980.

28. Sane S, Abdullah A, Boudreau DA, Autenried RK, Gupta BK, Wang X, Wang H, Schlenker EH, Zhang D, Telleria $\mathrm{C}$ et al: Ubiquitin-like (UBX)-domain-containing protein, UBXN2A, promotes cell death by interfering with the p53-Mortalin interactions in colon cancer cells. Cell death \& disease 2014, 5:e1118.

29. Srivastava S, Vishwanathan V, Birje A, Sinha D, D'Silva P: Evolving paradigms on the interplay of mitochondrial Hsp70 chaperone system in cell survival and senescence. Crit Rev Biochem Mol Biol 2019, 54(6):517-536.

30. Xu M, Jin T, Chen L, Zhang X, Zhu G, Wang Q, Lin Z: Mortalin is a distinct bio-marker and prognostic factor in serous ovarian carcinoma. Gene 2019, 696:63-71.

31. Starenki D, Sosonkina N, Hong SK, Lloyd RV, Park Jl: Mortalin (GRP75/HSPA9) Promotes Survival and Proliferation of Thyroid Carcinoma Cells. Int J Mol Sci 2019, 20(9).

32. Meireles Da Costa N, Ribeiro Pinto LF, Nasciutti LE, Palumbo A, Jr.: The Prominent Role of HMGA Proteins in the Early Management of Gastrointestinal Cancers. Biomed Res Int 2019, 2019:2059516.

33. Lee S, Rauch J, Kolch W: Targeting MAPK Signaling in Cancer: Mechanisms of Drug Resistance and Sensitivity. Int J Mol Sci 2020, 21(3).

34. Hoang VT, Nyswaner K, Torres-Ayuso P, Brognard J: The protein kinase MAP3K19 phosphorylates MAP2Ks and thereby activates ERK and JNK kinases and increases viability of KRAS-mutant lung cancer cells. J Biol Chem 2020.

35. Zhou C, Huang Y, Chen Y, Xie Y, Wen H, Tan W, Wang C: miR-602 Mediates the RASSF1AVJNK Pathway, Thereby Promoting Postoperative Recurrence in Nude Mice with Liver Cancer. OncoTargets and therapy 2020, 13:6767-6776.

36. Jorgensen K, Davidson B, Florenes VA: Activation of $\mathrm{c}$-jun $\mathrm{N}$-terminal kinase is associated with cell proliferation and shorter relapse-free period in superficial spreading malignant melanoma. Modern Pathol 2006, 19(11):1446-1455.

37. Hagiwara S, Kudo M, Chung H, Ueshima K, Inoue T, Haji S, Watanabe T, Park A, Munakata H, Sakurai $\mathrm{T}$ : Activation of c-Jun $\mathrm{N}$-terminal kinase in non-cancerous liver tissue predicts a high risk of recurrence after hepatic resection for hepatocellular carcinoma. Hepatology research : the official journal of the Japan Society of Hepatology 2012, 42(4):394-400.

\section{Table}


Table 1. Multivariate analyses for recurrence-free survival by Cox regression model

\begin{tabular}{lccc}
\hline Parameters & \multicolumn{3}{c}{ Multivariate analysis } \\
\cline { 2 - 4 } & HR & 95\% CI & P \\
\hline Age & 1.658 & $0.769-3.575$ & 0.197 \\
Sex & 0.994 & $0.515-1.918$ & 0.986 \\
Smoking history & 1.100 & $0.804-1.50$ & 0.552 \\
& & 4 & \\
Stage & 1.688 & $0.848-3.360$ & 0.136 \\
HMGA1 expression & 2.486 & $1.249-4.948$ & 0.009 \\
GRP75 expression & 0.973 & $0.492-1.921$ & 0.936 \\
\hline
\end{tabular}

\section{Figures}

a

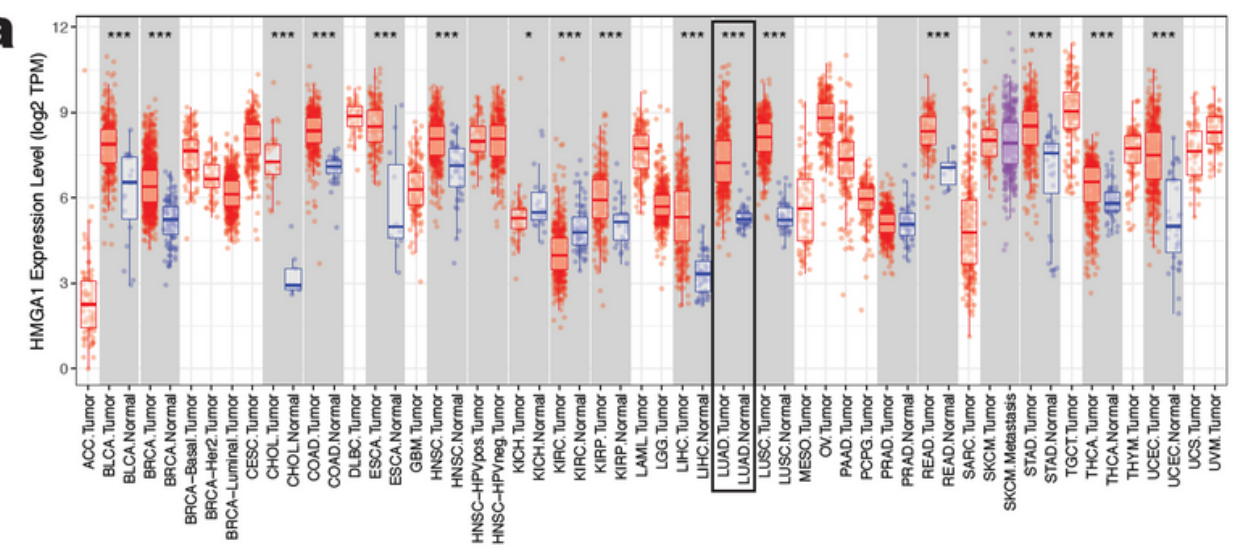

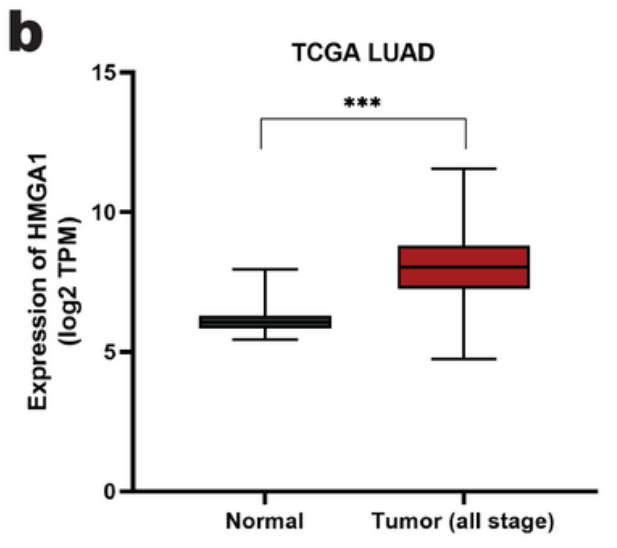

6
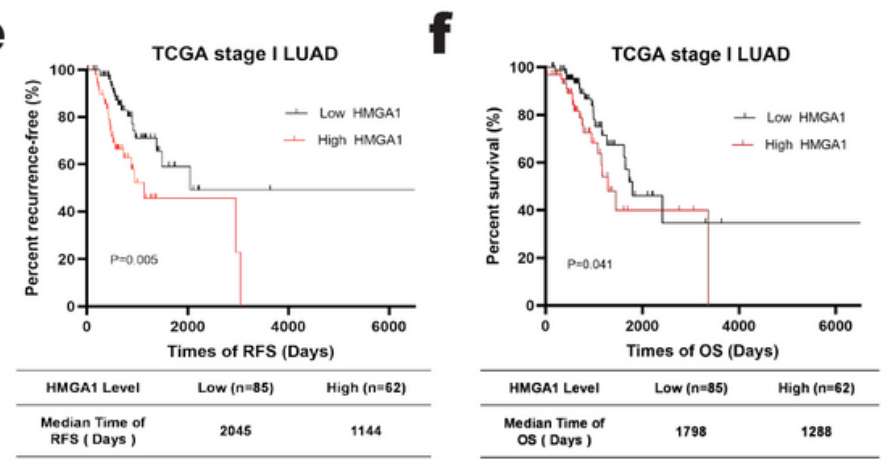

Figure 1

HMGA1 expression level was associated with recurrence and poor prognosis in Stage I LUAD. a HMGA1 mRNA expression in different cancers from The Cancer Genome Atlas (TCGA) datasets. TPM, Transcripts Per Million. b Expression of HMGA1 in all stage LUAD tumor samples compared with normal tissues. $c$ Expression level of HMGA1 in Stage I LUAD samples significantly higher than normal. $d$ The HMGA1 expression level was significantly associated with recurrence in stage I LUAD patients from the TCGA cohort. e The HMGA1 high-expression group had a significantly lower median time of RFS (Recurrencefree survival) rate than those in the HMGA1 low-expression group in stage I LUAD patients from the TCGA cohort. $f$ The HMGA1 high-expression group had a significantly lower OS (overall survival) rate than those 
in the HMGA1 low-expression group in stage I LUAD patients from the TCGA cohort. *, p < 0.05; ***, $p<$ 0.001 .

a
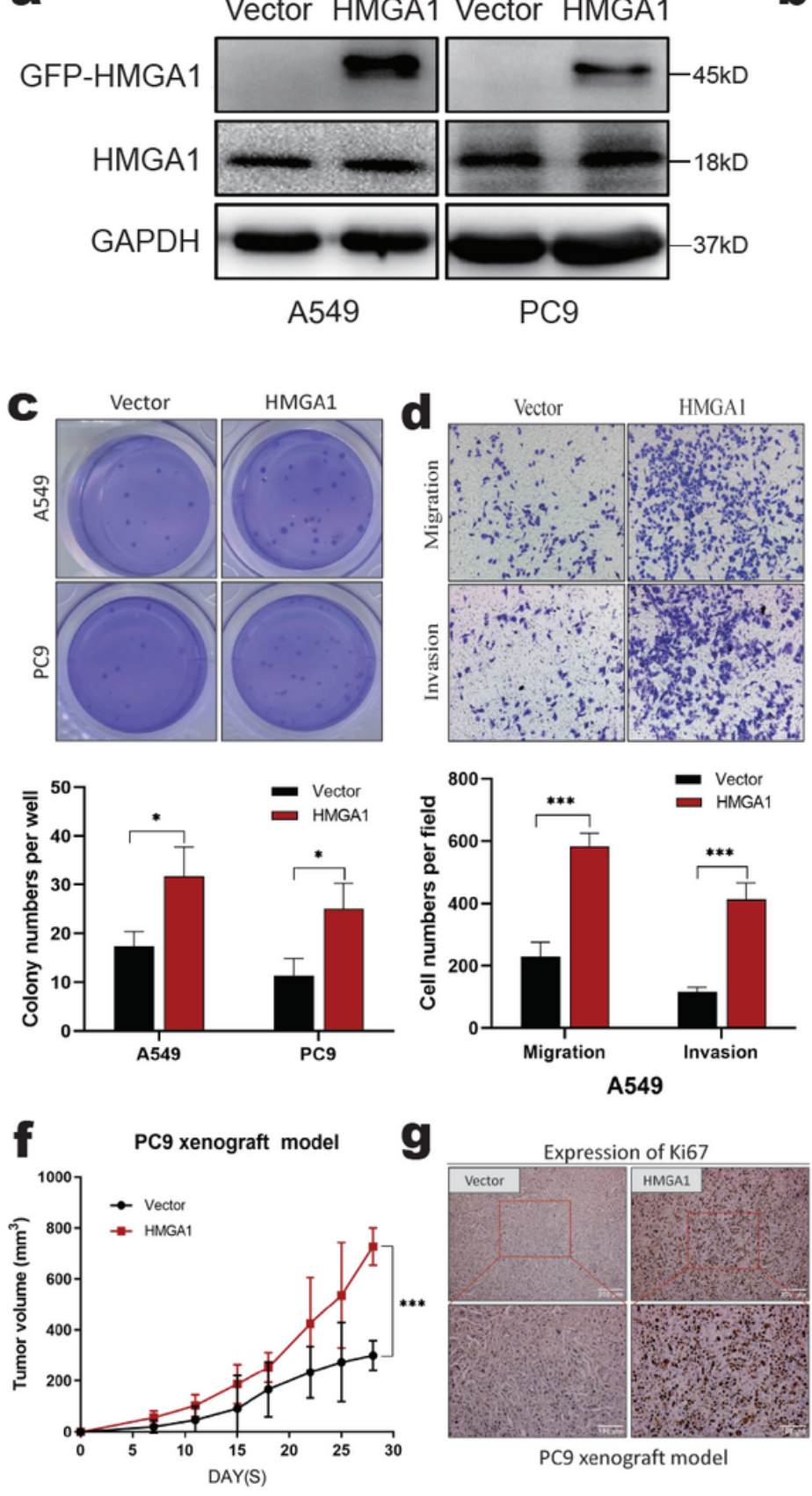

b
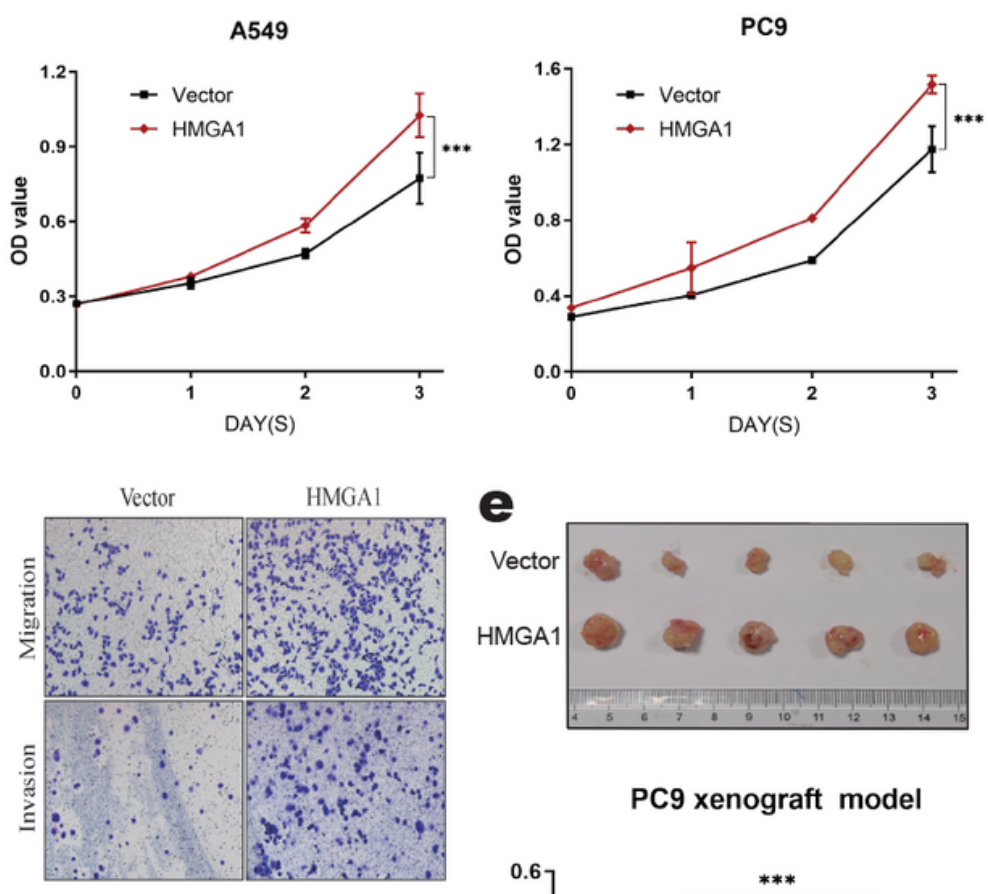

PC9 xenograft model

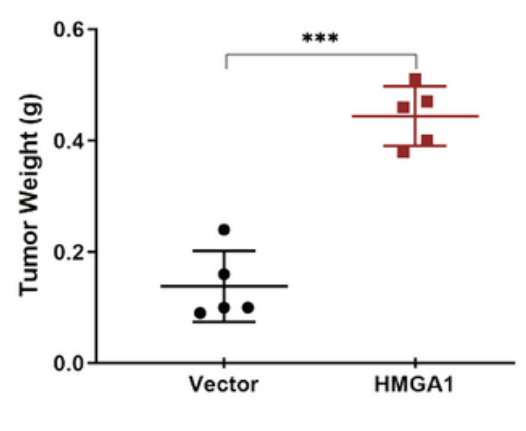

h A549 lung metastasis model
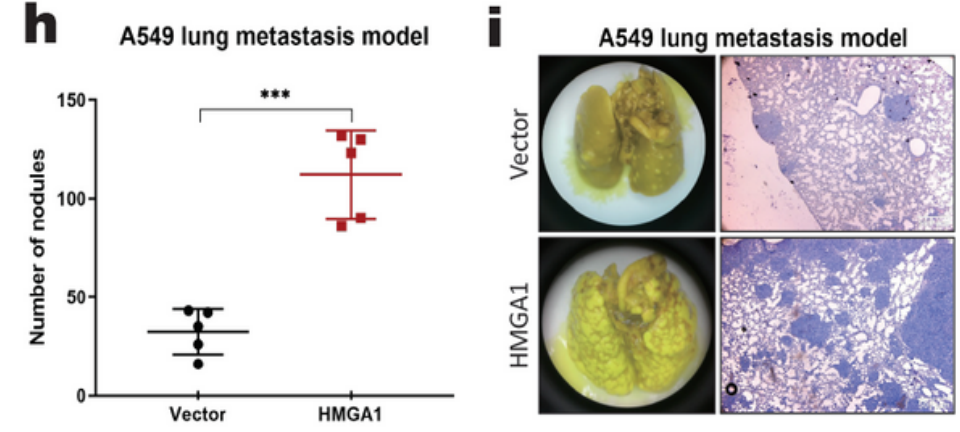

Figure 2

Overexpression of HMGA1 increases LUAD cell viability and metastasis. a Western blot analysis of the expression of HMGA1 in A549 and PC9 cells. Indicated cells were transfected with construct that expressing green fluorescent protein (GFP)-tagged HMGA1. After 72 hours of transfection, HMGA1 was detected using HMGA1 antibodies. b Overexpression of HMGA1 significantly stimulated LUAD cell viability using the CCK8 assays. c-d Overexpression of HMGA1 significantly stimulated Soft agar colony formation, migration and invasion of LUAD cells. Three independent experiments were conducted. Data were presented as the mean \pm S.D. e Weights and images of tumors from PC9 xenograft model $(n=5$ per 
group). f HMGA1 overexpression stimulated tumor growth in PC9 xenograft model. $g$ Immunohistochemistry assay showed that HMGA1 overexpression increased cell proliferation marker protein Ki67-positive LUAD cells. The tumor tissues were from PC9 xenograft model. h-i Overexpression of HMGA1 significantly promoted lung metastases. Images of metastatic lung nodules in nude mice $(\mathrm{n}=5$ per group) and HE staining were performed after four weeks by tail-vein injection of A549 LUAD cells. * $p$ $<0.05 ; * \star, p<0.01 ; * \star *, p<0.001$.

a

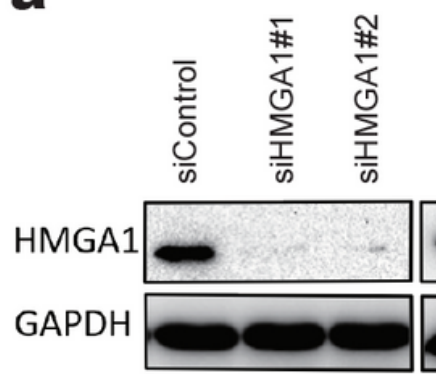

A549

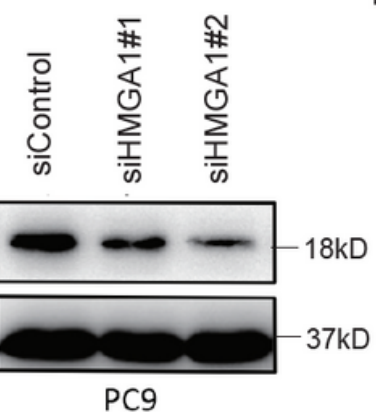

PC9 b

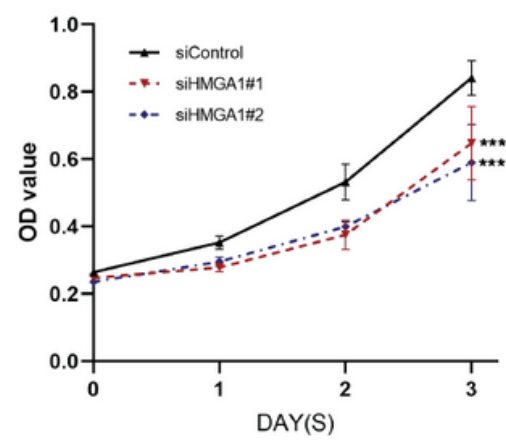

PC9

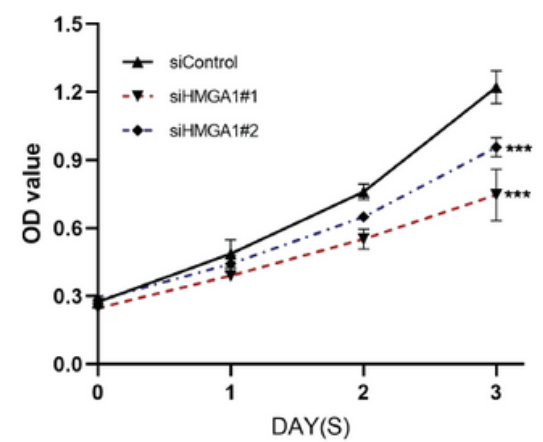

6

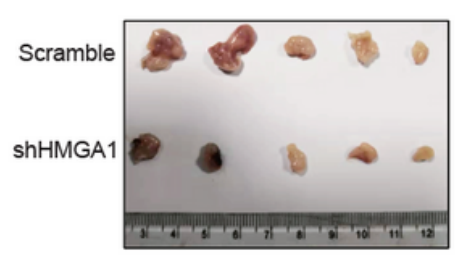

PC9 xenograft model
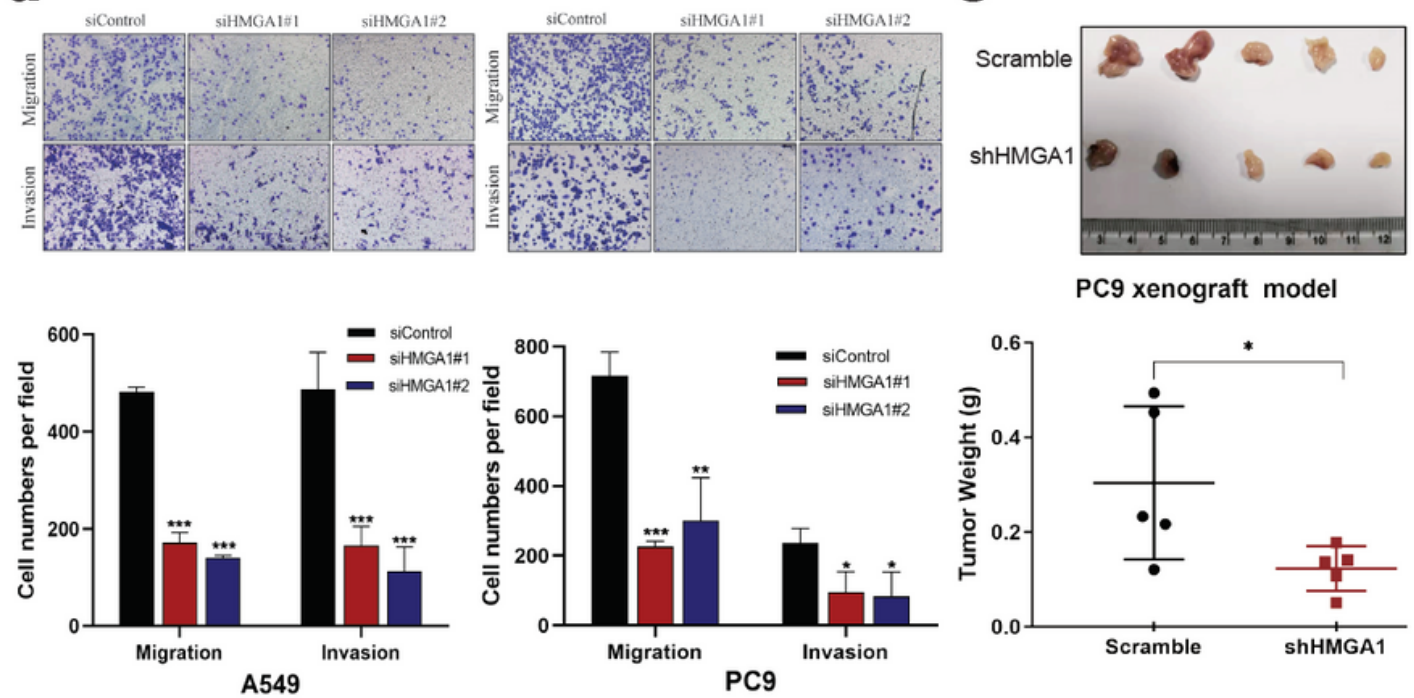

h A549 lung metastasis model

g

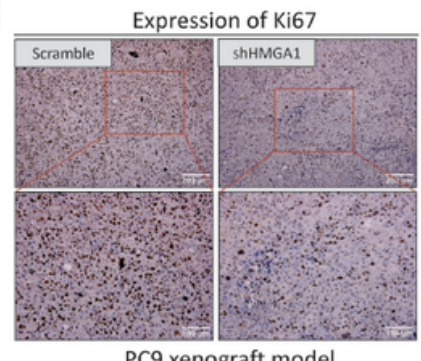

PC9 xenograft model
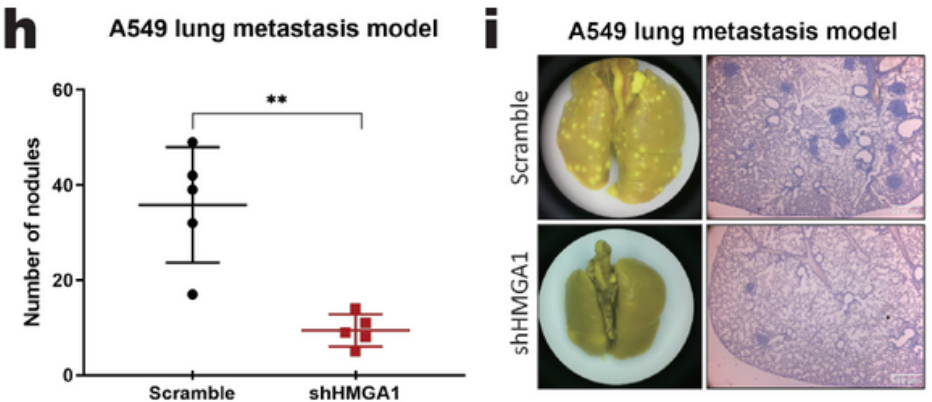

\section{Figure 3}

Inhibition of HMGA1 inhibits LUAD progression. a HMGA1 siRNA treatment significantly inhibited HMGA1 expression in LUAD cells. Indicated cells were transfected with siRNAs of HMGA1. After 72 hours of transfection, cells were subjected to Western blot analysis. b CCK8 assays showed that inhibition of 
HMGA1 significantly inhibits LUAD cells viability. c-d Inhibition of HMGA1 significantly suppressed Soft agar colony formation, migration and invasion of LUAD cells. Three independent experiments were conducted. Data were presented as the mean \pm S.D. e Weight and image of tumors showed that inhibition of HMGA1 significantly inhibited tumor size in PC9 xenograft model ( $n=5$ per group). $f$ The tumor volumetime curves had shown that HMGA1 down-expression inhibited tumor growth. g Immunohistochemistry assay showed that inhibition of HMGA1 significantly decreased Ki67-positive cells in LUAD tissues from PC9 xenograft model. h-i HMGA1 down-expression inhibited lung metastases. Images of metastatic lung nodules in nude mice ( $n=5$ per group) and HE staining were performed after four weeks by tail-vein injection of A549 LUAD cells. *, $p<0.05 ; * \star, p<0.01 ; * \star *, p<0.001$. 
a

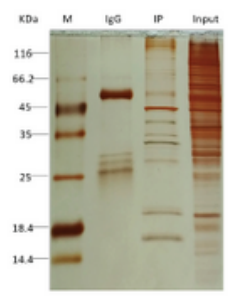

d

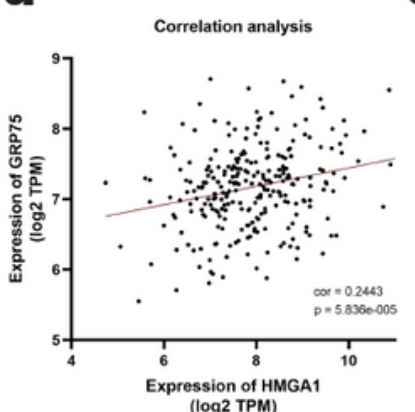

$\mathbf{h}$
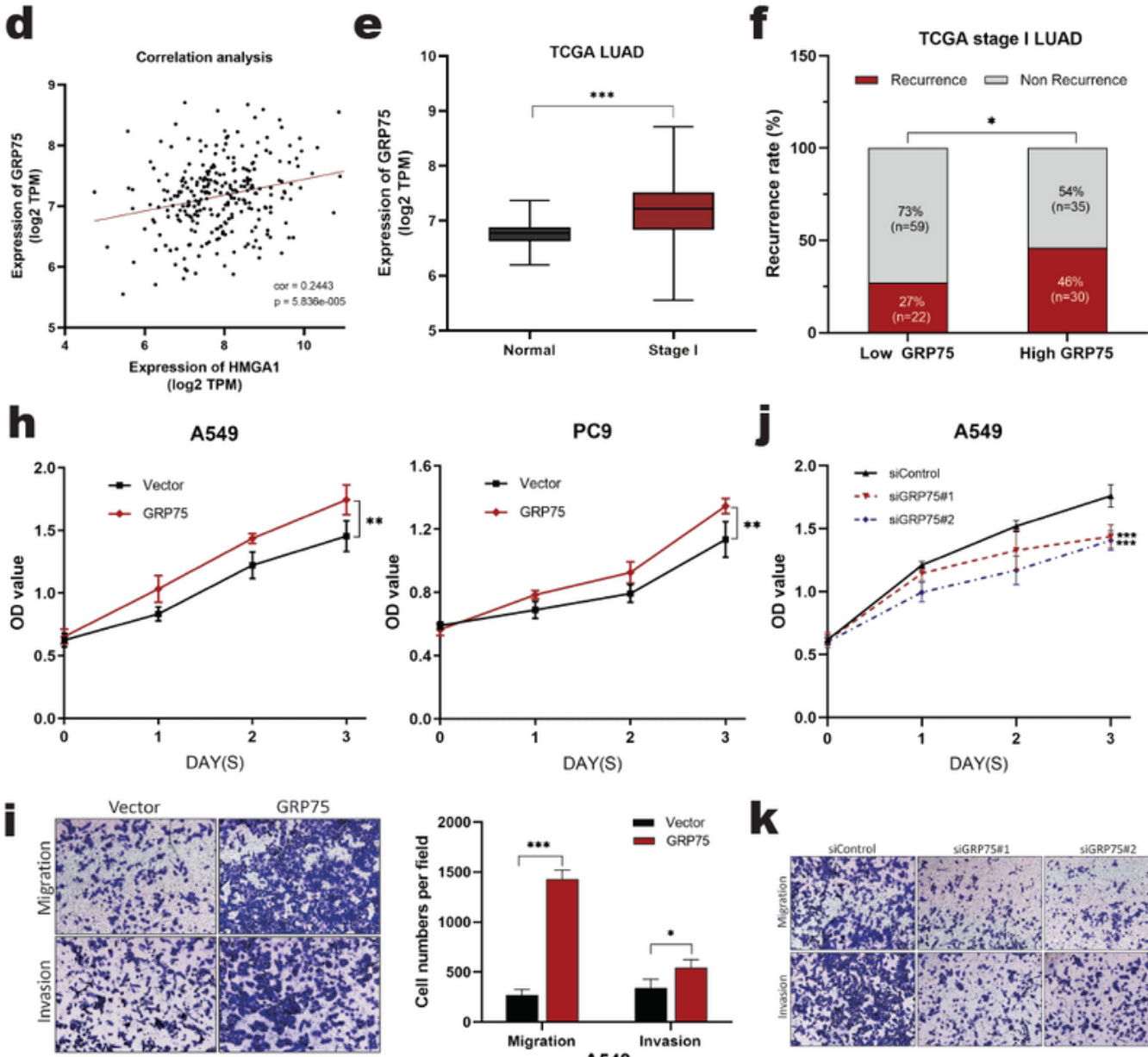

j

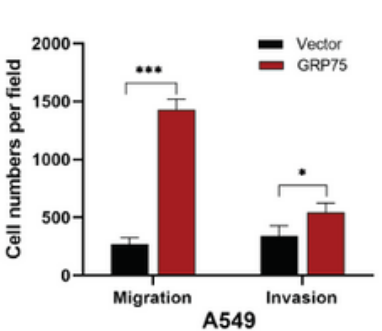

$\mathbf{k}$
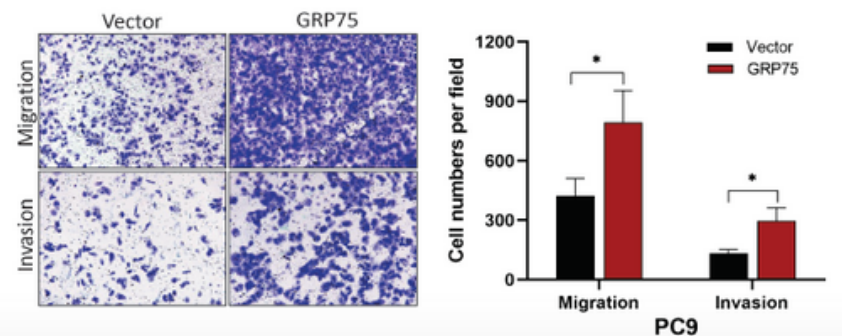
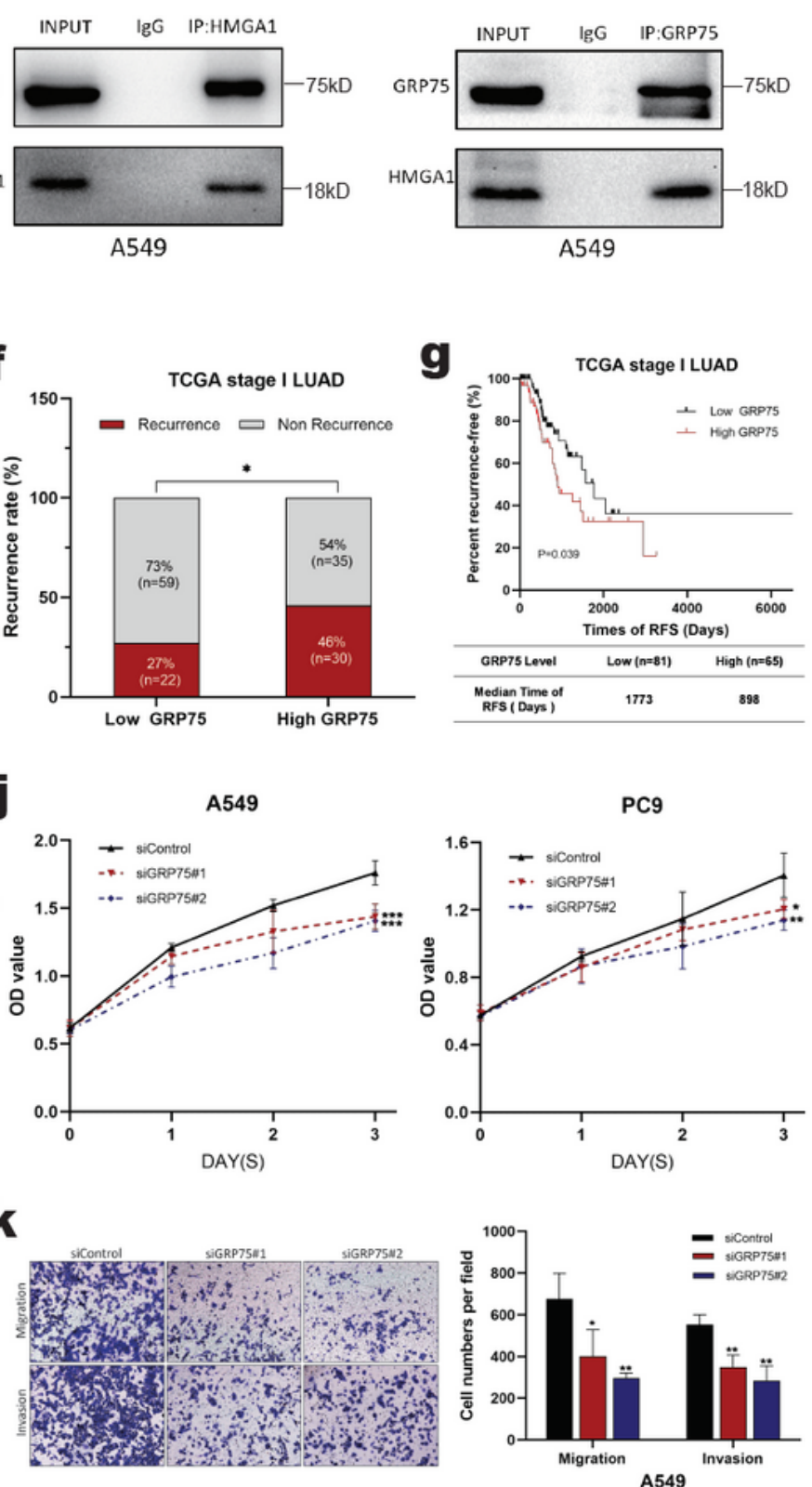

PC9
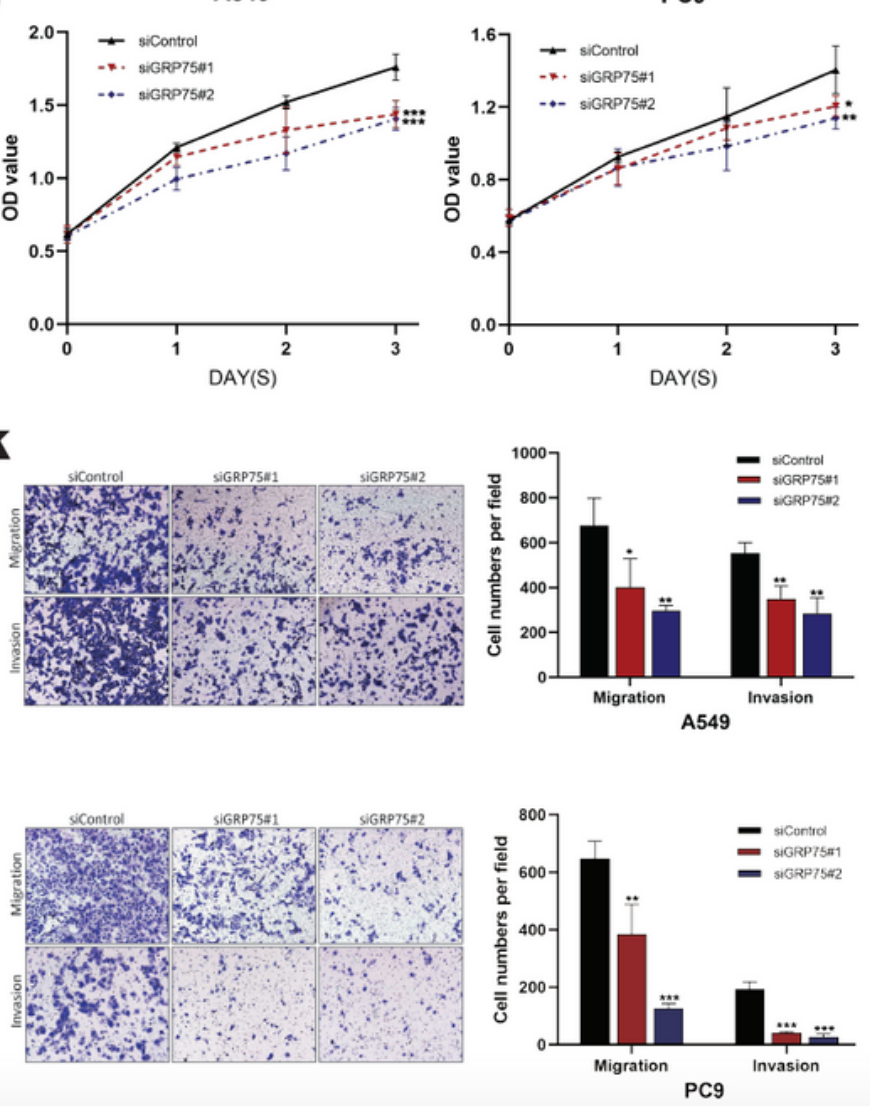

Figure 4

GRP75 interacts with HMGA1 and plays oncogenic role in LUAD. a Silver staining image of proteins that interact with HMGA1 in LUAD cells. A549 cells were transfected with vector or HMGA1 expression plasmid for 72 hours. Cell lysate was immunoprecipitated with anti-HMGA1 and IgG, and the proteins interact with HMGA1 were separated with SDS-PAGE, and stained with silver. b Venn plot of differential gene expression analysis. The proteins interact with HMGA1 and IgG were detected by mass 
spectrometry. c Co-IP assays were performed to confirm the interaction of GRP75 with HMGA1 in A549 cells with anti-HMGA1 or anti-GRP75 antibody. $\mathrm{d}$ TCGA data set analysis showed that GRP75 protein expression level is positively correlated with HMGA1 protein expression level in stage I LUAD. TPM, Transcripts Per Million. e TCGA data set analysis showed that expression of GRP75 was significantly increased in stage I LUAD samples compared with normal tissues. f TCGA data set analysis showed the expression level of GRP75 was closely associated with recurrence in stage I LUAD patients. g TCGA data set analysis showed the expression level of GRP75 was closely associated with shorter recurrence-free survival time in stage I LUAD patients. h CCK8 analysis showed that GRP75 stimulated LUAD cells viability. $i$ Transwell assays showed that GRP75 stimulated migration and invasion of LUAD cells. j CCK8 assay showed that inhibition of GRP75 significantly inhibits LUAD cells viability. k Inhibition of GRP75 significantly suppressed migration and invasion of LUAD cells. Three independent experiments were conducted. Data were presented as the mean \pm S.D. *, $p<0.05 ; * \star, p<0.01 ; * \star *, p<0.001$. 

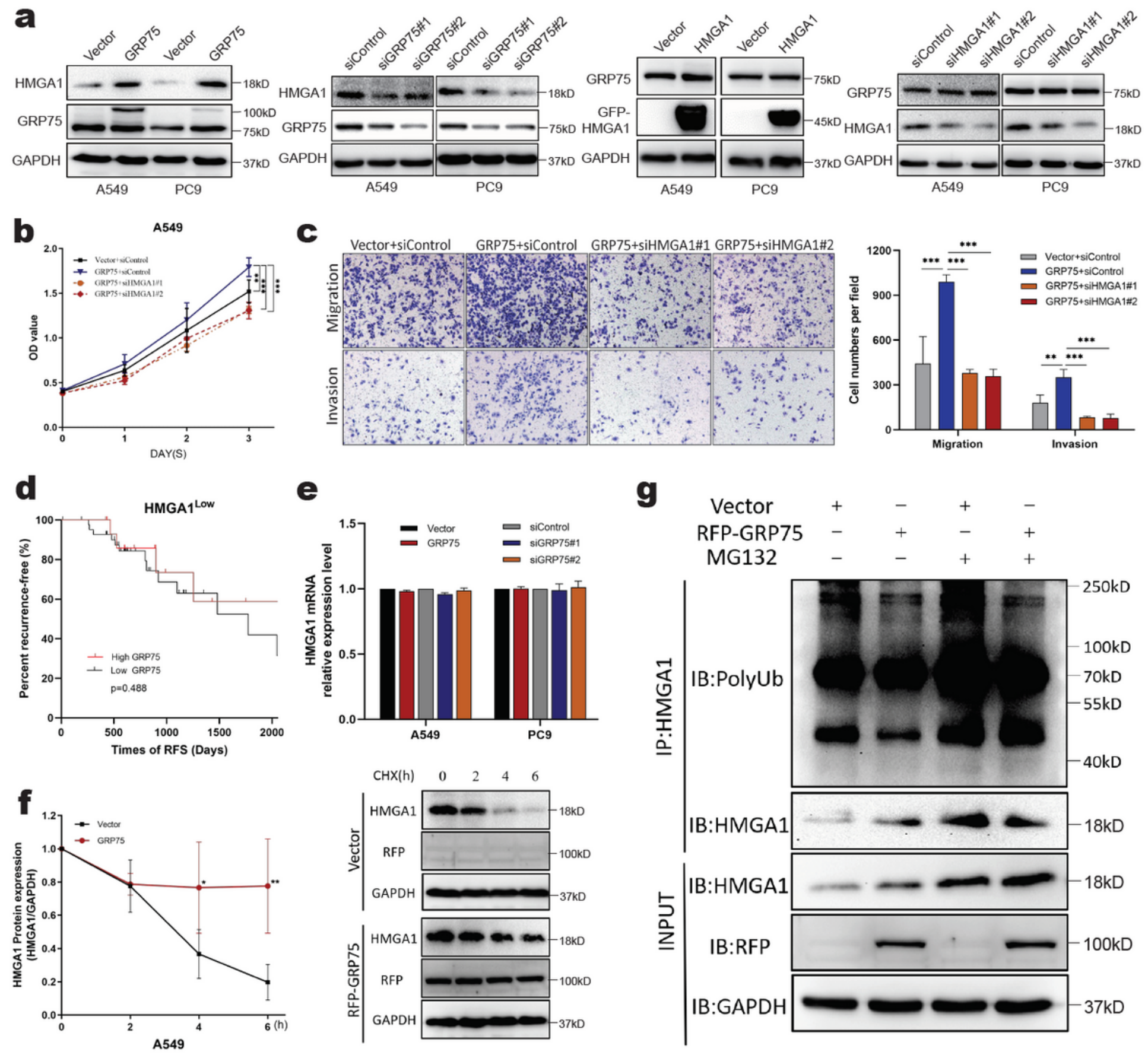

Figure 5

HMGA1 is the functional downstream target of GRP75 in LUAD cells. a GRP75 positively regulated HMGA1 expression in LUAD cells, but HMGA1 did not affect GRP75 expression. Indicated cells were transfected with indicated plasmids or siRNA for 72 hours, then subjected to Western blot analysis. Bands corresponding to RFP-GRP75 fusion protein runs with apparent molecular weights of about 100 kD. b CCK8 assay showed that silencing of HMGA1 blocked overexpression of GRP75-induced LUAD cell viability stimulation. c Transwell assay showed that silencing of HMGA1 blocked overexpression of GRP75-induced stimulation of migration and invasion. d TCGA data set analysis showed there was no correlation between the expression level of GRP75 and recurrence-free survival (RFS) time in stage I LUAD 
patients with low expression of HMGA1. e GRP75 did not affect HMGA1 mRNA expression in LUAD cells. Indicated cells were transfected with indicated plasmid or siRNA for 72 hours, then cells were subjected to qRT-PCR analysis. $f$ Overexpression of GRP75 significantly prolonged the half-life time of HMGA1 in LUAD cells. A549 cells with or without overexpressing GRP75 were treated with CHX $(100 \mu \mathrm{g} / \mathrm{ml}, \mathrm{CST})$ for the indicated times. Western blot analysis and Grayscale analysis of HMGA1 protein levels were showed. g Overexpression of GRP75 inhibited HMGA1 ubiquitination in LUAD cells. A549 cells were transfected

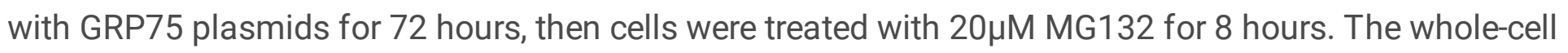
lysates were subjected to immunoprecipitation with HMGA1 antibody and Western blot with anti-Ub antibody to detect ubiquitylated HMGA1. Three independent experiments were conducted. Data were presented as the mean \pm S.D. *, $\mathrm{p}<0.05 ; * \star, \mathrm{p}<0.01 ;{ }^{* \star}, \mathrm{p}<0.001$. 
a

b
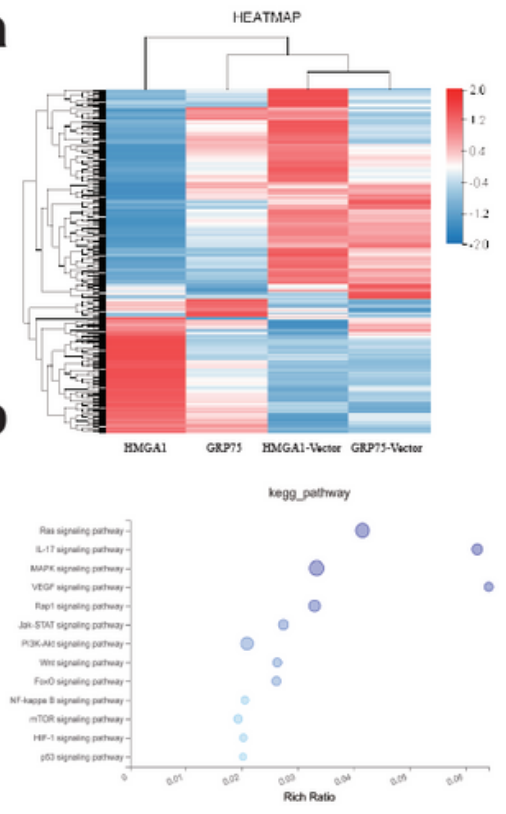

A549

e

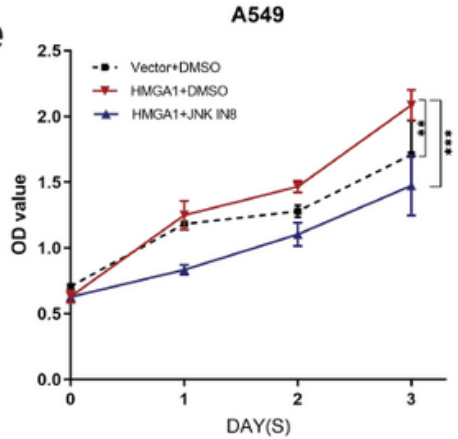

f
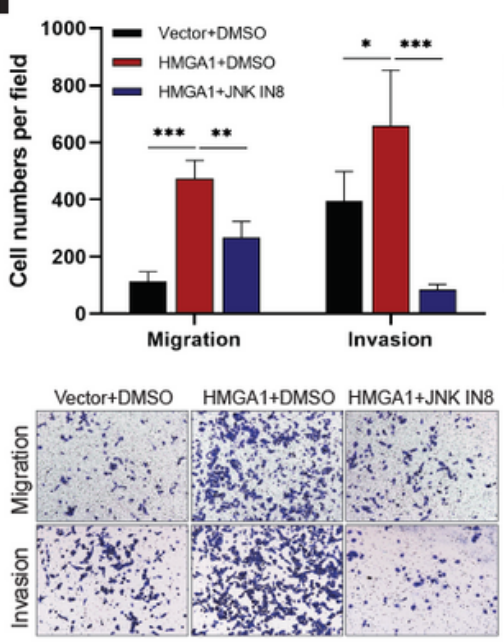

C

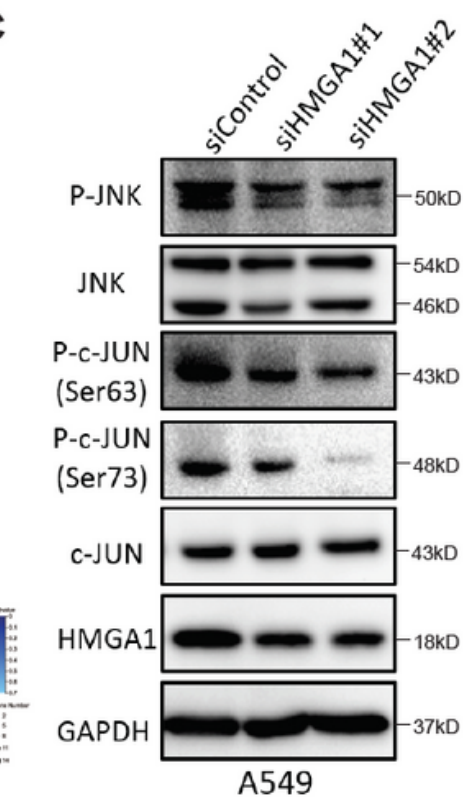

A549
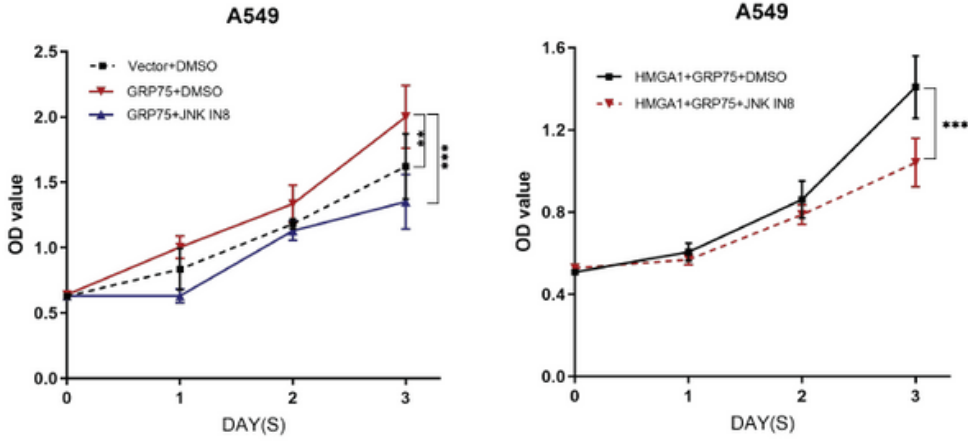

g
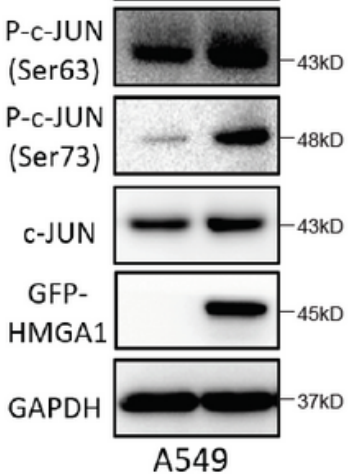

GAPDH

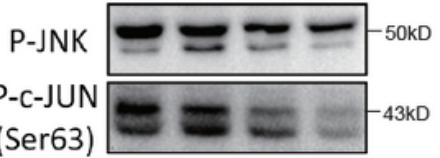

P-C-JUN

(Ser73)

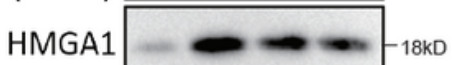

GRP75

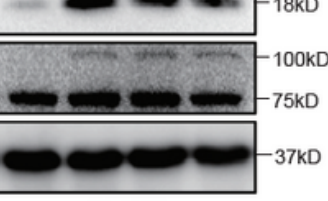

A549

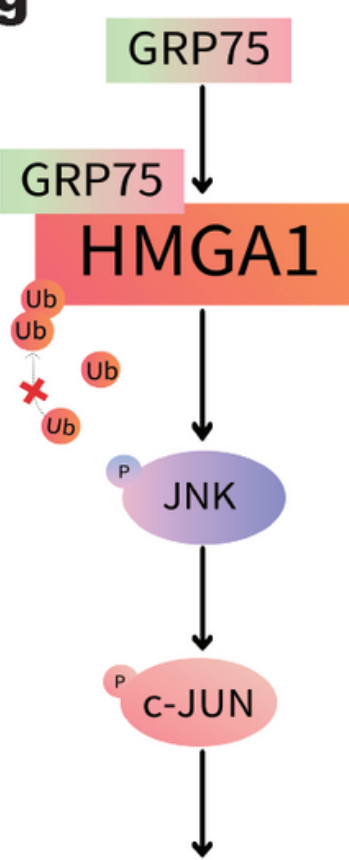

Tumor progression

\section{Figure 6}

GRP75/HMGA1 axis stimulates LUAD progression by activating JNK/c-JUN signaling pathway. a Heatmap shown genes that expression level was affected by overexpressing HMGA1 or GRP75 in LUAD cells. A549 cells were transfected with indicated plasmid for 72 hours, then subjected to mRNA sequencing. b MAPK signaling was significantly affected by GRP75/HMGA1 axis in LUAD cells. KEGG signal pathway enrichment analysis was performed using genes that similarly affected by HMGA1 and GRP75 overexpression. c Western blot analysis showed that HMGA1 positively regulated the 
phosphorylation of JNK(Thr183/Tyr185), c-JUN (Ser63) and c-JUN (Ser73) in A549 cells. Cells were transfected with HMGA1 plasmids or HMGA1 siRNAs for 72 hours, then subjected to Western blot analysis. $d$ Inhibition of HMGA1 blocked GRP75 overexpression induced phosphorylation of JNK and cJUN in A549 cells. Cells were transfected with HMGA1 plasmids or HMGA1 siRNAs for 72 hours, then subjected to Western blot analysis. e CCK8 analysis showed that JNK inhibitor treatment blocked overexpression HMGA1 or/and GRP75-induced cell viability stimulation. $f$ Transwell analysis showed that JNK inhibitor treatment blocked overexpression of HMGA1 or/and GRP75-induced stimulation of cell migration and invasion. Indicated cells were transfected with indicated plasmid for 48 hours, then cells were treated with $1 \mu \mathrm{M}$ JNK IN8 (JNK inhibitor) for 24 hours. g A schematic model for the progression mechanism of LUAD by GRP75/HMGA1 axis in LUAD cells. Three independent experiments were conducted. Data were presented as the mean \pm S.D. $*, p<0.05 ; * \star, p<0.01 ; * \star *, p<0.001$.

\section{Supplementary Files}

This is a list of supplementary files associated with this preprint. Click to download.

- Additionalfile1Tables1.pdf

- Additionalfile2FigS1.pdf

- Additionalfile3Figs2.pdf

- OriginalData.docx

- TranswellrawData.pdf 